\title{
"Fluid-Fluid" levels on a Primary Leiomyosarcoma of the Bone: First Case Report to the Best of Our Knowledge
}

\author{
López Soriano*1, Palomar Ramos ${ }^{2}$, Patricia Fraga Rivas ${ }^{3}$, Cebada Chaparro ${ }^{4}$ and Crespo Rodríguez ${ }^{5}$ \\ ${ }^{1}$ Elsa. Hospital Clínico San Carlos, Spain \\ ${ }^{2}$ Jorge Hospital Clínico San Carlos, Spain \\ ${ }^{3}$ Hospital del Henares. Av. de Marie Curie 2, Spain \\ ${ }^{4}$ Elena. Hospital Clínico San Carlos, Spain \\ ${ }^{5}$ Hospital Clínico San Carlos, Spain
}

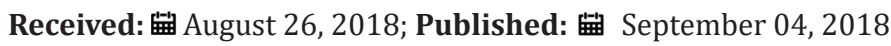

*Corresponding author: Elsa López Soriano, Elsa. Hospital Clínico San Carlos, Calle Profesor Martín Lagos, 28040, Madrid, Spain

\begin{abstract}
Primary leiomyosarcoma (LMS) of the bone is a very rare tumor with smooth muscle differentiation on immunohistochemical studies. Radiologically, LMS appears as an osteolytic lesion with aggressive features but no pathognomonic radiological findings that helps making the diagnosis. We report one case of primary LMS of the femur with fluid-fluid levels (FFLs) on the MRI, the first case to our knowledge. We consider we should include primary LMS of the bone in the differential diagnosis of "FFL" lesions.

Keywords: Leiomyosarcoma; Fluid Fluid Levels; MRI

Abbreviations: LMS: Leiomyosarcoma; FFL: Fluid-Fluid Levels; MRI: Magnetic Renonance Imaging; WHO: World Health Organization; ABC: Aneurismal Bone Cyst; WI: Weigthed Images
\end{abstract}

\section{Introduction}

LMS is a malignant tumor comprising of spindle-shaped cells with distinctive smooth muscle cell features [1]. They are approximately $7 \%$ to $10 \%$ of all soft tissue sarcoma [2]. Most of these tumors occur frequently in uterus and soft-tissues and more rarely in bone [3-5]. The first description of a primary bone LMS in a long bone was given in 1965 [1]. Since then, to the best of our knowledge, around 130 cases of primary LMS of bone have been reported, mostly small case reports that have focused on its histological diagnosis [4]. The clinical behavior of osseous LMS is generally aggressive. Most of the published studies of LMS of the bone have reported poor prognosis with a $35 \%$ overall survival rate [6].

LMS diagnosis is established by the presence of morphologically typical spindle cells with smooth muscle differentiation and the positivity of a smooth muscle actin and other muscle markers on tumor cells, such as desmin and h-caldesmon [7]. Furthermore, the specific characteristic of primary bone LMS is a solitary osteolytic lesion with indistinct margins and cortical destruction with the absence of either osteoid or chondroid matrix [8,9]. Since its recognition as a distinct entity, to our knowledge no sizeable series has examined its imaging features. Furthermore, only a few studies about primary LMS of the bone with emphasis on MRI findings have been published [10]. We present the clinical features, radiological findings and pathological description of a primary LMS originating from the distal femur in a middle-aged lady. Best to our knowledge, this is the first case report of "FFLs" on MRI of a primary LMS of the bone.

\section{Case Report}

40-year-old women presenting with one-month pain on the left knee. She denied fever, history of trauma or any other symptoms. She had no pathological medical history. Blood tests were performed, showing hemoglobin level $12.3 \mathrm{~g} / \mathrm{dl}$, white blood cell count 5300/L with normal differential, creatinine level $0.45 \mathrm{mg} /$ $\mathrm{dl}$, glucose $93 \mathrm{mg} / \mathrm{dl}$. Radiography of the left knee revealed bone lesion in the metaphysis and epiphysis of the distal left femur. It was a permeative lytic lesion. Neither periosteal reaction nor mineralization matrix was shown. An uncertain thinning of the medial cortical was present. CT scan showed osteolytic lesion in the distal metaphysis of the left femur with thinning of the anteromedial cortical. It had an eccentric medullary location on 
the axial plane. No periosteal reaction or evidence of soft tissue mass was shown. MRI without and with paramagnetic contrast revealed a bone lesion in the distal metaphysis of the left femur. It was predominantly hypo-isointense on T1-weigthed images (WI) and had very heterogeneous high signal intensity on T2-WI. It had a central oval area of $2 \mathrm{~cm}$ that had a cystic behavior with FFLs. The medial cortical was overpassed by the tumor (Figures 1-3).

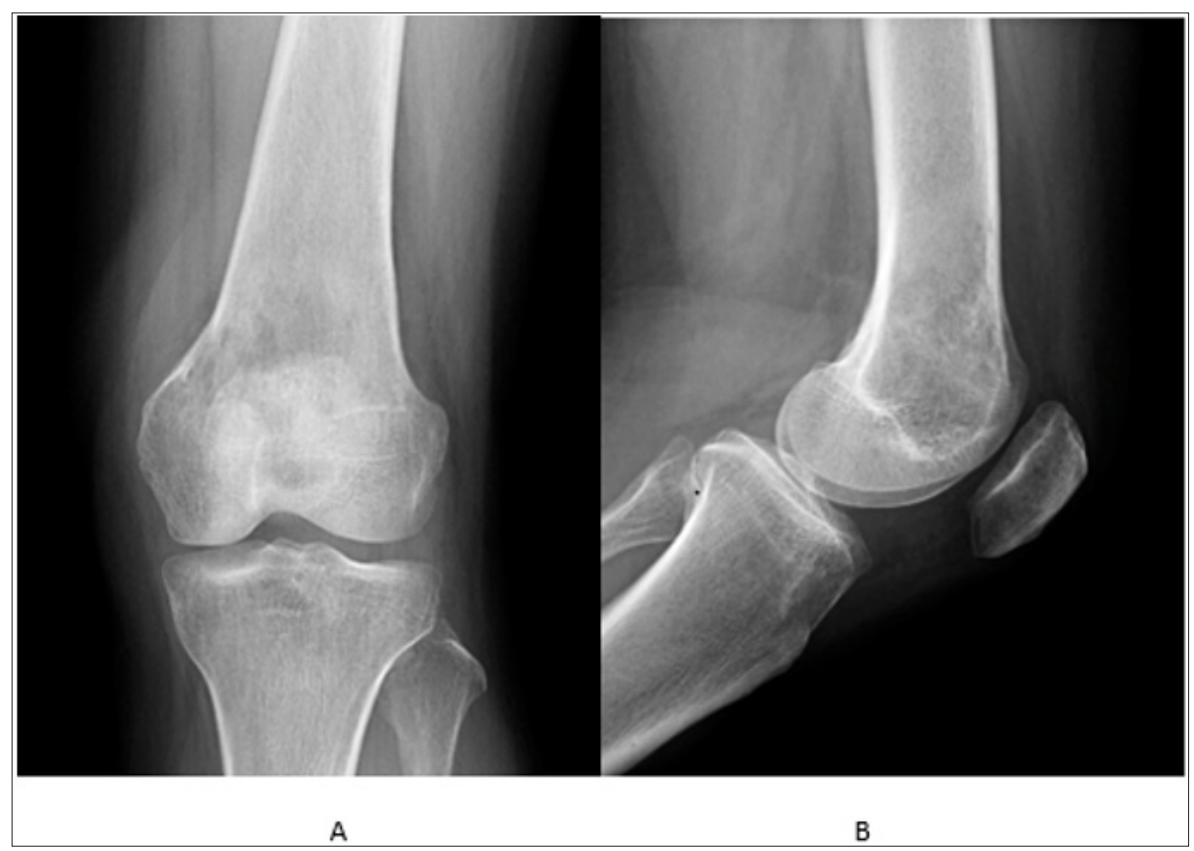

Figure 1: Anteroposterior radiograph of left knee.

Note:

A. lateral radiograph of left knee

B. show ill-defined, predominantly osteolytic lesion of left distal femur with endosteal thinning.

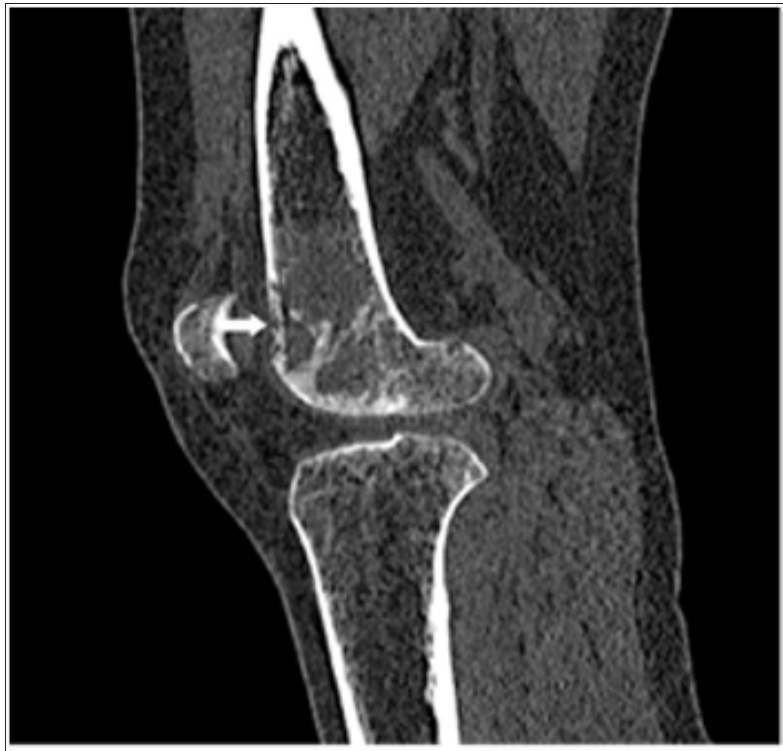

A

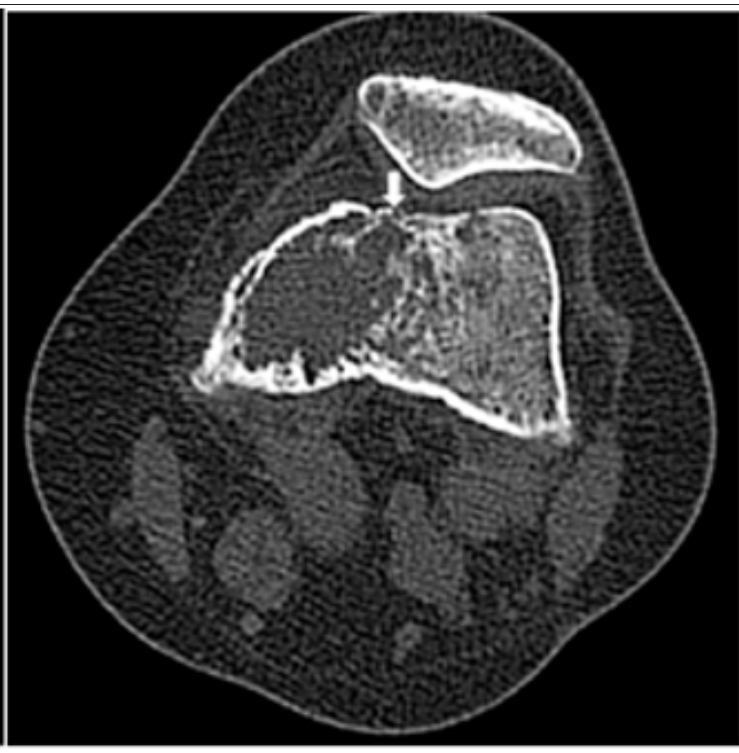

B

Figure 2: Sagittal CT Anterior cortical breaching is visible on sagittal view.

Note:

A. axial CT (b) of the left distal femur show metaphyseal osteolytic lesion with extension to either the diaphysis and the epiphysis (juxtaarticular portion of the long bone).

B. Neither matrix mineralization nor accompanying sclerosis were found. 


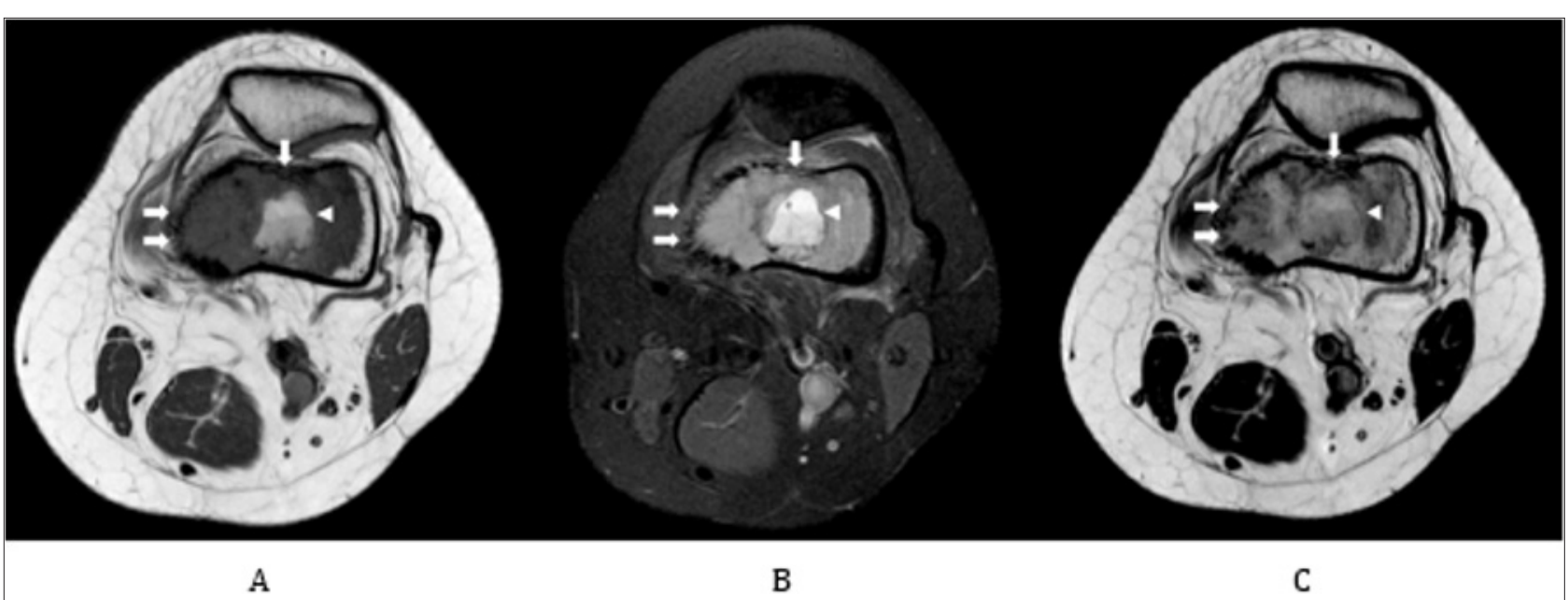

Figure 3: Axial MRI.

Note:

A. Axial DP fat sat image shows a bone lesion on the distal epiphysis of the left femur, moderately hyperintense, that overpass the cortical and a central oval area with fluid-fluid levels.

B. Axial T1 shows the hypointense tumor with a central oval area with fluid-fluid levels.

C. Axial T1 post contrast shows an extensive diffuse and heterogeneous contrast enhancement of the tumor.

On post-contrast $\mathrm{T} 1$ fat sat $\mathrm{WI}$, extensive diffuse and heterogeneous contrast enhancement hard tissue was shown. Central oval area did not present enhancement on the post-contrast images. The patient was referred to our institution for an evaluation by an Osteoncology Multidisciplinary Team, composed of, at least, an orthopedist, an oncologist, a radiologist, a pathologist, a radiotherapist, a physiatrist, a palliative therapist, and a nuclear medicine physician. The team suggested a biopsy of the bone lesion. PET-TC showed a FDG deposit on a lytic lesion on the distal epiphysis of the left femur with SUV max 9.7, in relation with the primary tumor. No other FDG deposits were found. There was a light subtle deposit in the uterus, probably functional (premenstrual phase) with SUVmax 2.6. The multidisciplinary team reviewed the patient clinical condition, the imaging findings, and the results of the biopsy, suggesting neoadjuvant chemotherapy and surgery of the bone LMS.

\section{Discussion}

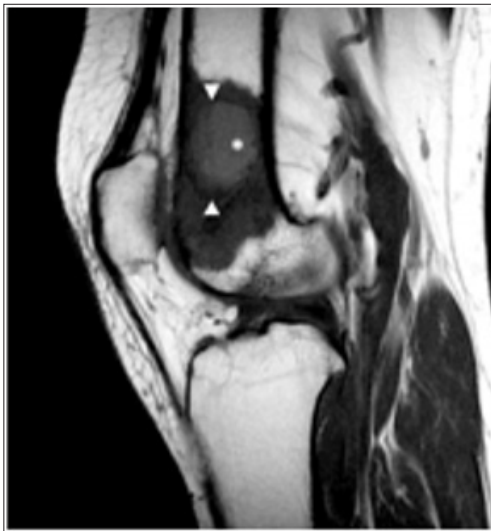

A

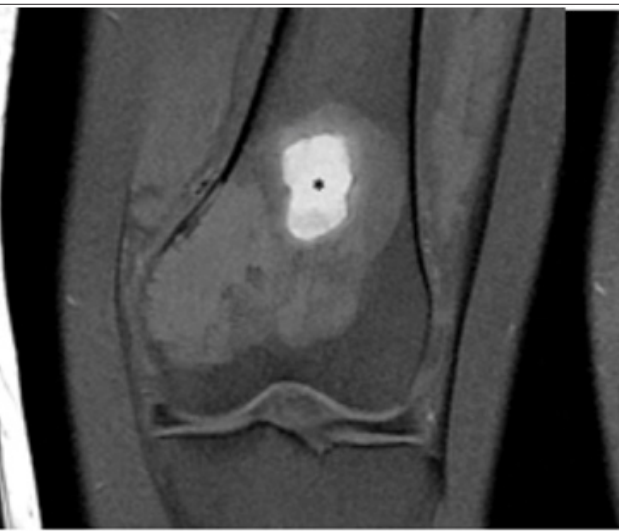

B

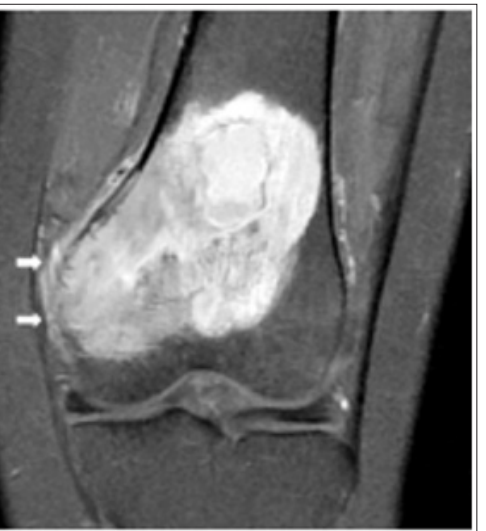

C

Figure 4: A: Sagital T1 MRI, an hypo-isointense lesion occupying juxtaarticular epiphysis and metaphysis of the femur with a central oval area. FFLs are not clearly seen on this images. B: Coronal T1 fat sat shows a hypointense tumor with an oval hyperintense area with fluid- fluid levels. C: Coronal T1 fat sat postcontrast: intense enhancement is shown in the lesion and in soft tissues adyacent to the medial cortical.

LMSs are malignant soft tissue tumors with smooth muscle differentiation apparent with immunohistochemical or electron microscope studies (WHO) $[4,8,11]$. They are approximately $7 \%$ to
$10 \%$ of all soft tissue sarcoma [2]. LMS can be further subclassified into cutaneous, gastrointestinal, uterine and somatic lesions $[3,4]$. In most cases, somatic LMS occurs primarily in the retroperitoneum, 
the soft tissue of the extremities, the blood vessels, or rarely, in the bone [5]. When a LMS appears in the skeleton the possibility of a metastasis should be considered. Primary LMS of the bone is fairly uncommon, with less than $0.7 \%$ incidence of all primary malignant bone tumors [1,3]. There is a wide age distribution, with a preference for those middle-aged or older. Although LMS has been reported in patients as young as 9 years old, the mean age for diagnosis of this tumor in case series studies is about 50 years $[4,11]$. Primary LMS of bone most frequently occurs in the long bones of the lower extremities, particularly distal femur [4,5,1113] (Figure 4).

Histologic features of primary LMS of the bone does not differ from those of LMS found elsewhere in the body [5]. It is just one of the variants of spindle cell sarcoma of bone characterized by the expression of desmin and other markers indicating a significant element of smooth muscle in the tumor, without osteoid production [9].

Immunohistochemical staining allows the diagnosis by demonstrating the presence of smooth muscle markers and immunoreactivity in tumor cells. Immunohistochemical findings could be a diagnostic tool, since several tumors of the bone (conventional and nonconventional osteosarcoma, malignant fibrous histiocytoma, giant cell tumor, chondroblastoma) could express smooth-muscle actin. H-Caldesmon seems to be a more specific marker [13]. On plain radiographs, LMS of bone appears as an osteolytic lesion with indistinct margins and moth-eaten or permeative pattern $[4,8,11,15]$. No mineralization matrix can be recognized. In addition to osteolysis, bone lesions show various degrees of aggressiveness, as the lack of a sclerotic rim and cortical breakthrough [4,8,11] (Figure 5).

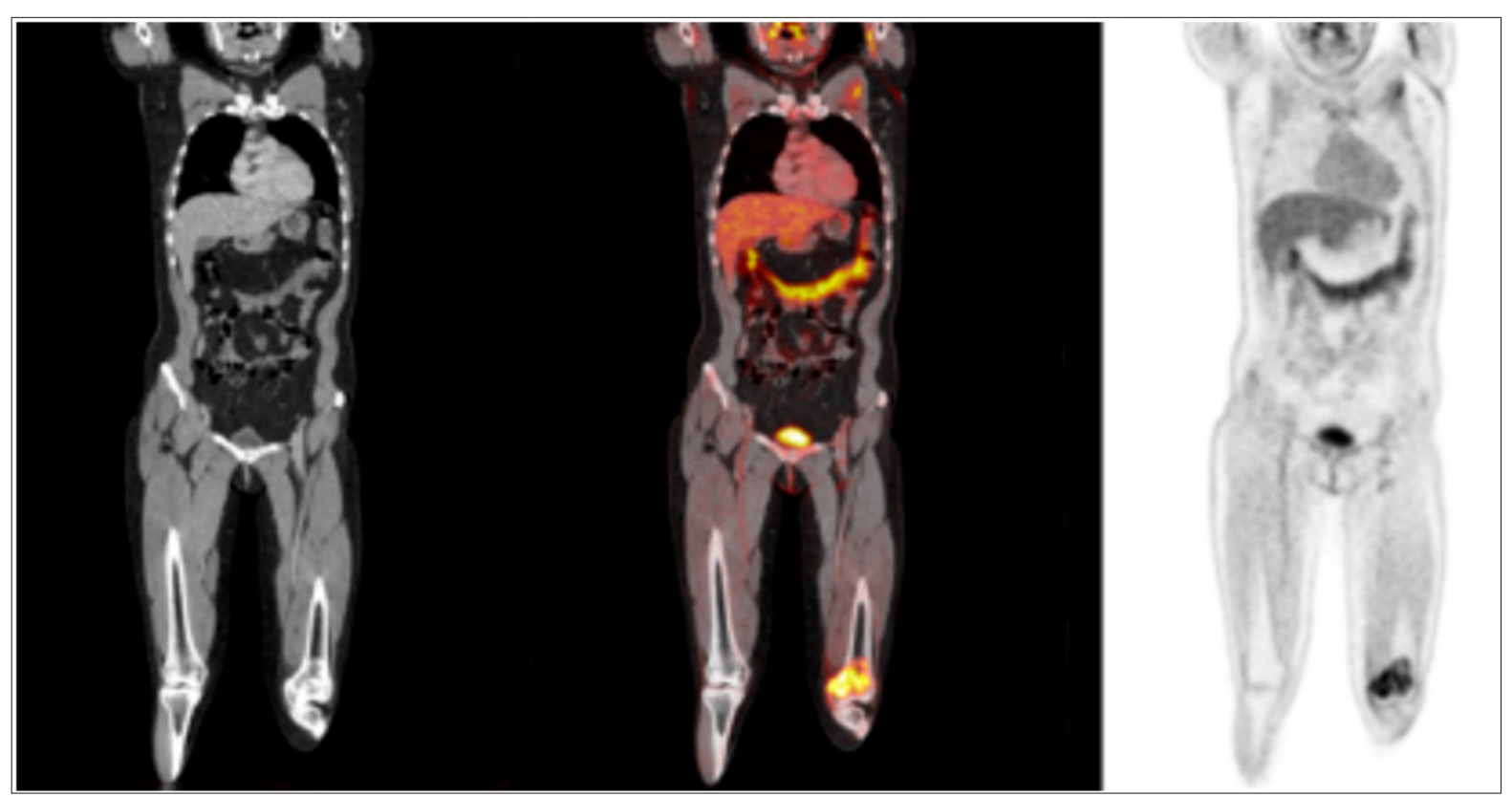

Figure 5: PET-CT: showed a FDG deposit on a lytic lesion on the distal epiphysismetaphysis of the left femur with SUV max 9.7, in relation with primary tumor.

Description of the MRI findings of a primary osseous LMS is limited. LMS of the bone is usually hypointense on T1-WI and showed heterogeneous signal intensity on T2-WI [10]. Sundaram et al. also pointed out a striking feature in long bones, that was its exceptional length of this primary malignant tumor of bone, unlike the typical appearance of metastatic disease [10]. FFLs can appear when substances with different densities are contained within a cystic or compartmentalized structure [16]. The presence of these levels in a bone lesion suggests internal bleeding, followed by stratification of the blood components according to their density [16-18] but they can also be seen in malignant tumors with liquefactive necrosis. This necrosis may follow chemotherapy or radiotherapy [19]. These levels can be observed in simple radiographs and tomographic studies, but MRI is the best method to diagnose them [18]. The levels are depicted when imaging is performed in a gravity dependent plane [19].
The upper level is usually hyperintense in relation to the lower level in the power sequence in $\mathrm{T} 2$, because the latter usually contains the cellular elements. However, in the sequence enhanced in T1, the appearance varies depending on the stage of the lesion, presenting a signal increase in the upper level when the bleeding is subacute, presumably due to the presence of extracellular methemoglobin [20]. In the formulation of a differential diagnosis, we should start combining clinical information, location and radiographic appearance of the lesion. However, differential diagnosis of an osteolytic lesion with permeative pattern without mineralization matrix beyond LMS of the bone, includes other common entities as metastasis, myeloma, pleomorphic sarcoma and classically is characteristic of osteomyelitis and round cell tumors (lymphoma, Ewing's sarcoma and neuroblastoma) [21]. Our case demonstrated a central cystic area with FFLs. Since the initial observation of FFLs on CT and MRI of aneurysmal bone cyst (ABC) [22,23], they have been identified in many different types of tumors (Figure 6). 


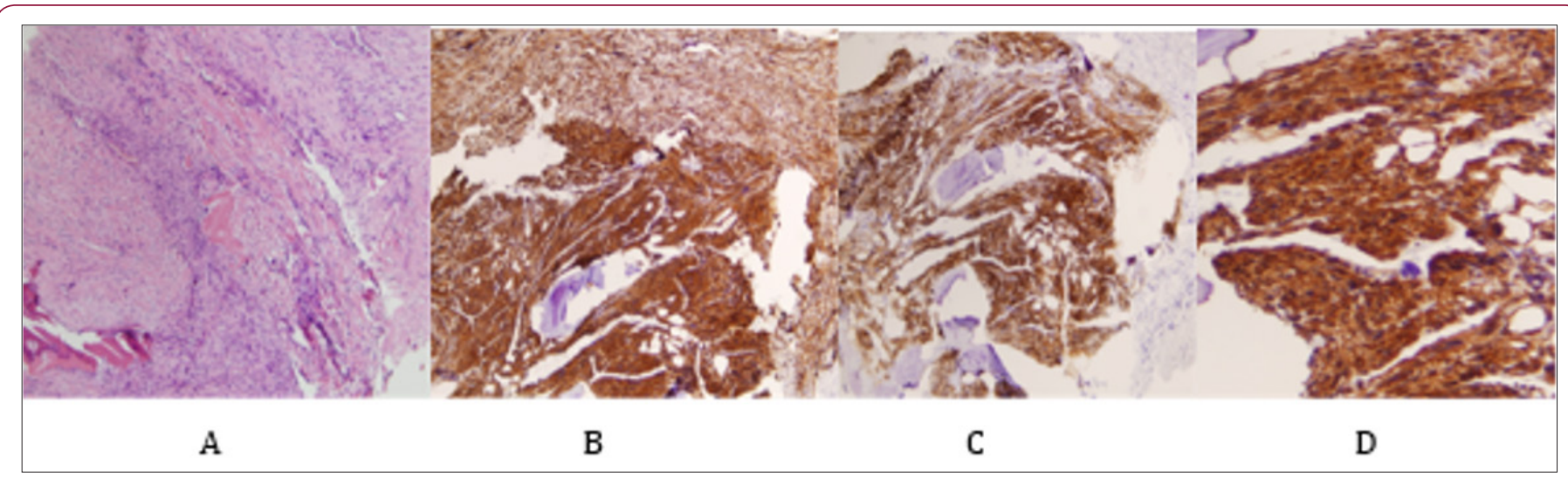

Figure 6: Histology from a surgical biopsy: fascicles of pleomorphic spindle cells with marked atypia. The tumor lacks osteoid. Immunohistochemically, the cells show positive staining with smooth muscle actin, calponina and caldemon but not desmin. The number of mitosis was 8/10 high-power fields. No necrosis was found.

Note:

A. Fascicles of pleomorphic spindle cells with marked atypia (H-E, x100).

B. Positive staining with smooth muscle actin.

C. Positive staining with caldesmon.

D. Positive staining with calponina.

Overall prevalence of FFLs in bone tumors reported by O'Donnell et al. [24] was $11.2 \%$ in a series of 738 consecutive patients referred with focal lesions of bone. The extent of FFLs within a focal bone lesion appears to be inversely related to the degree of malignancy [25]. If at least $2 / 3$ of the lesion shows FFL change, $89 \%$ of diagnoses are benign. Differential diagnosis in a bone lesion with FFLs would include common diagnosis as $\mathrm{ABC}$ and telangiectatic osteosarcoma, less common diagnoses as giant cell tumor, unicameral bone cyst [26], chondroblastoma [27] and rare diagnoses as brown tumor (hyperparathyroidism) [28], osteoblastoma [29] and fibrous dysplasia [30]. Primary ABC is ruled out in our case by aggressive radiological features on plain films, as it usually presents as a sharply demarcated expansive, lytic lesion with a thin and uninterrupted cortical margin on plain films [24]. Differentiation of telangiectatic osteosarcoma and ABC based on visualization of FFLs either on CT or MRI can be problematic [22]. The malignant appearance of telangiectatic osteosarcoma could already be appreciated on plain radiographs, with a permeating pattern of cortical and trabecular bone destruction or a periosteal reaction with Codman's triangles [31] (Figure 7).

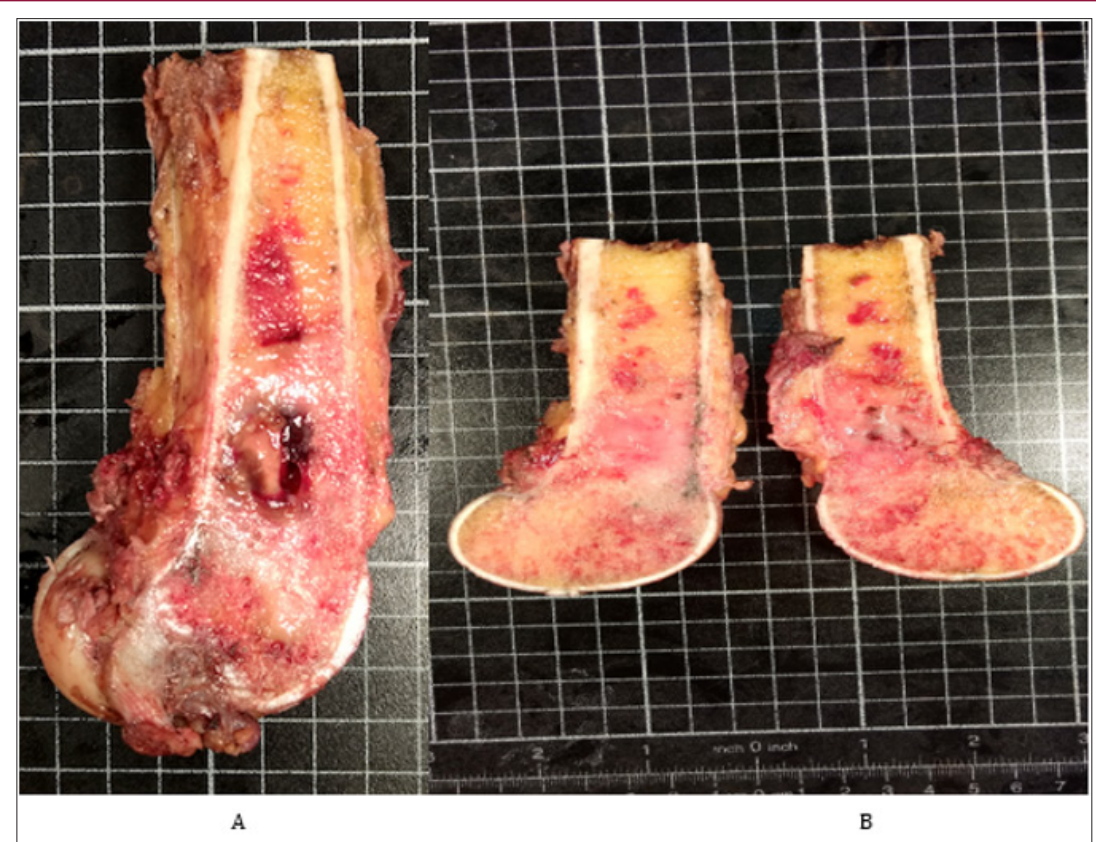

Figure 7: surgical specimen shows the tumor in the metaphysis with extension to the epiphysis and invasion of soft tissues. Note:

A. A central cystic area is shown.

B. The cut surface appears white and inhomogeneous. 
Giant cell tumors of bone, also known as osteoclastomas, are relatively common bone tumors, usually benign, that typically arise from the metaphysis of long bones and extend into the epiphysis. Although FFLs can be seen on MRI when a secondary $\mathrm{ABC}$ component is present, heterogeneous high signal with areas of low signal intensity due to hemosiderin or fibrosis [32] are characteristic. Other primary bone lesion with a secondary $\mathrm{ABC}$ formation $[23,24,27,32,33]$ can be ruled out because of location (osteoblastoma), age (chondroblastoma) or clinical presentation (fibrous dysplasia, brown tumor) do not match in this particular case. To the best of our knowledge, this is the first report of FFLs of a primary osseous LMS. After recognition that FFLs occurs in LMS of the bone, this particular entity should be included in the differential diagnosis. Furthermore, a better understanding of FFLs as a nonspecific finding seen in a wide range of bone and soft-tissue tumors [16] improved the diagnostic approach. In this particular case, after revision of the patient clinical condition, the imaging findings, and the results of the biopsy, the multidisciplinary team suggested neoadjuvant chemotherapy and surgery of the bone LMS. It is generally agreed that early resection of the tumor seems to be the best choice of definitive treatment and the clinical outcome of LMS of bone is simply affected by whether surgery with a wide margin is possible or not $[34,35]$. The effectiveness of combination chemotherapy or radiotherapy is still unclear. Moreover, the current chemotherapy regimens had little impact on the improvement of survival unlike other types of chemo- sensitive primary bone sarcoma, such as osteosarcoma in children and young adults [35].

\section{Conclusion}

Primary bone LMS is a rare tumor and it is a diagnosis of exclusion. Radiological findings show aggressive lytic lesions, but it does not allow differential diagnosis with other aggressive bone tumors. Diagnosis requires histology and immunohistochemistry staining. As far as we know, this is the first case described in the literature with the radiological finding of FFLs. We believe that it should be included in the differential diagnosis of bone tumors with this radiological sign.

\section{References}

1. Evans DM, Sanerkin NG (1965) Primary leiomyosarcoma of bone. J Pathol Bacteriol 90(1): 348-350.

2. Siegel RL, Miller KD, Jemal A (2016) Cancer statistics, 2016. CA: A Cancer Journal for Clinicians 66(1): 7-30.

3. Somerhausen NDS, Cin PD (2002) Smooth muscle tumours. In: Fletcher CDM, Mertens F, Unni KK, (Eds) Pathology and Genetics of Tumours of Soft Tissue and Bone, IARC Press, Lyon, France, pp. 127-134.

4. Berlin O, Angervall L, Kindblom LG, Berlin IC, Stener B (1987) Primary leiomyosarcoma of bone. A clinical, radiographic, pathologic-anatomic, and prognostic study of 16 cases. Skeletal Radiol 16(5): 364-376.

5. Abraham JA, Weaver MJ, Hornick JL, Zurakowski David, Ready John E (2012) Outcomes and prognostic factors for a consecutive case series of 115 patients with somatic leiomyosarcoma. J Bone Joint Surg Am 94(8): 736-744.

6. Stramare R, Orsatti G, Attar S (2016) Imaging features, differential diagnosis and management of leiomyosarcomas: Case series and review of the literature. J Canc Sci Ther 8: 84-91.

7. Fletcher CDM, Unni KK, Mertens F (2002) Pathology and Genetics of Tumors of Soft Tissue and Bone. World Health Organization. In: Fletcher
CDM, Unni KK, Mertens F (Eds); International Agency for Research on Cancer, IARC Press, Lyon, France, p. 427.

8. Myers JL, Arocho J, Bernreuter TW, Dunham W, Mazur MT (1991) Leiomyosarcoma of bone: A clinicopathologic, immunohistochemical and ultrastructural study of five cases. Cancer 67(4): 1051-1060.

9. Brewer P, Sumathi V, Grimer RJ, Carter SR, Tillman RM, et al. (2012) Primary Leiomyosarcoma of Bone: Analysis of Prognosis. Hindawi Publishing Corporation Sarcoma. 2012: 636849.

10. Sundaram M, Akduman I, White LM, McDonald DJ, Kandel R, et al. (1999) Primary leiomyosarcoma of bone. AJR Am J Roentgenol 172: 771-776.

11. HannachiSassi S, Khattech R, Dhouib R, Rameh S, Mrad K, et al. (1999) Primary bone leiomyosarcoma. Anatomoclinical, immunohistochemical and ultrastructural study. Rev Chir Orthop Reparatrice Appar Mot 85(8): 865-870.

12. Jundt G, Moll C, Nidecker A, Schilt R, Remagen W (1994) Primary leiomyosarcoma of bone: Report of eight cases. Hum Pathol 25(11): 1205-1212.

13. Watanabe K, Tajino T, Sekiguchi M, Suzuki T (1996) h-Caldesmon as a specific marker for smooth muscle tumors. Comparison with other smooth muscle markers in bone tumors. Am J ClinPathol 113(5): 663668.

14. Goto T, Ishida T, Motoi N, Satoshi Yokokura, Hirotaka Kawano, et al. (2002) Primary leiomyosarcoma of the femur. J Orthop Sci 7(2): 267273.

15. Endo K, Takahashi M, Matsui Y, Hasegawa T, Yasui N (2005) Minimally Invasive Taskforce of the American Association of Hip and Knee Surgeons. Bone tumor of the distal femur in a 67 year-old woman. Clin Orthop 440: 262-268.

16. Tsai JC, Dalinka MK, Fallon MD, Zlatkin MB, Kressel HY (1990) Fluid-fluid level: A nonspecific finding in tumors of bone and soft tissue. Radiology 175(3): 779-782.

17. Frenzel L, Javier RM, Eichler F, Zollner G, Sibilia J (2010) Multiple fluidfilled bone metastases. Joint Bone Spine 77(2): 171-173.

18. Bladt O, Demaerel P, Catry F, Van Breuseghem I, Ballaux F, et al. (2004) Multiple vertebral fluid-fluid levels. Skeletal Radiol 33(11): 660-662.

19. Wang XL, Gielen JL, Salgado R, Delrue F, De Schepper AMA (2004) Soft tissue aneurysmal bone cyst. Skeletal Radiol 33(8): 477-480.

20. Alyas F, Saifuddin A (2008) Fluid-fluid levels in bone neoplasms: Variation of T1-weighted signal intensity of the superior to inferior layers--diagnostic significance on magnetic resonance imaging. Eur Radiol 18(11): 2642-2651.

21. Atalar H, Gunay C, Yildiz Y, Saglik Y (2008) Primary leiomyosarcoma of bone: A report on three patients. Clin Imaging 32(4): 321-325.

22. Hudson T (1984) Fluid levels in aneurismal bone cysts: A CT feature. AJR 141(5): 1001-1004.

23. Hertzanu Y, Mendelsohn D, Gottschalk F (1984) Aneurysmal bone cyst of the calcaneus. Radiology 151(1): 51-52.

24. O’Donnell P, Saifuddin A (2004) The prevalence and diagnostic significance of fluid-fluid levels in focal lesions of bone. Skeletal Radiol 33(6): 330-336.

25. Van Dyck P, Vanhoenacker FM, Vogel J, Venstermans C, Kroon HM, et al. (2006) Prevalence, extension and characteristics of fluid-fluid levels in bone and soft tissue tumors. EurRadiol 16(12): 2644-2651.

26. Margau R, Babyn P, Cole W, Smith C, Lee F (2000) MR imaging of simple bone cysts in children: Not so simple. Pediatr Radiol 30(8): 551-557.

27. Davila JA (2004) Chondroblastoma of the hands and feet. Skeletal Radiol 33(10): 582-587.

28. Davies AM, Evans N, Mangham C, Grimer R (2001) MR imaging of brown tumor with fluid fluid levels: A report of three cases. EurRadiol 11(8): 1445-1449. 
29. Vilanova JC, Dolz JL, Maestro de Leon JL, Aparicio A, Aldoma J, et al. (1998) MR imaging of a malignant schwannoma and an osteoblastoma with fluid-fluid levels. Report of two new cases. EurRadiol 8: 1359-1362.

30. Gibson MJ, Middlemiss JH (1971) Fibrous dysplasia of bone. Br J Radiol 44(517): 1-13.

31. Vanel D, Tcheng S, Contesso G, Zafrani B, Kalifa C, et al. (1987) The radiological appearances of telangiectatic osteosarcoma. A study of 14 cases. Skeletal Radiol 16(3): 196-200.

32. Pereira HM, Marchiori E, Severo A (2014) Magnetic resonance imaging aspects of giant-cell tumours of bone. J Med Imaging RadiatOncol 58(6): 674-678.

ISSN: 2574-1241

DOI: $10.26717 / B J S T R .2018 .08 .001687$

López Soriano. Biomed J Sci \& Tech Res

(C) (i) This work is licensed under Creative

Submission Link: https://biomedres.us/submit-manuscript.php
33. Davies AM, Cassar Pullicino VN, Grimer RJ (1992) The incidence and significance of fluid fluid levels on computed tomography of osseous lesions. Br J Radiol 65(771): 193-198.

34. Gielen JLMA, De Schepper AMA, Vanhoenacker FM, Parizel PM, Wang XL, et al. (2004) Accuracy of MRI in characterization of soft tissue tumors and tumor-like lesions. A prospective study in 548 patients. EurRadiol 14(12): 2320-2330.

35. Mori T, Nakayama R, Endo M, Hiroaki Hiraga, Masato Tomita, et al. (2016) Forty-eight cases of leiomyosarcoma of bone in Japan: A multicenter study from the Japanese musculoskeletal oncology group. J SurgOncol Sep 114(4): 495-500.

BIOMEDICAL
RESEARCHES $\quad \begin{aligned} & \text { Assets of Publishing with us } \\ & \text { - Global archiving of articles } \\ & \text { - Immediate, unrestricted online access }\end{aligned}$

